\title{
Erratum to: Nonlinear optical absorption and NIR to blue conversion in highly stable polymeric dye rod
}

\author{
I. M. Azzouz • A. Salah
}

(C) Springer-Verlag Berlin Heidelberg 2013

\section{Erratum to: Appl Phys B (2012) 108:469-474 DOI 10.1007/s00340-012-4915-y}

The corresponding author would like to apologize for the mistakes appeared in the article and request the following rectifications.

1. The following corrected values for lasers should be used throughout the article:

Excitation sources were N2 laser (337 nm) and $808 \mathrm{~nm}$ diode laser (cw and pulsed modes).

2. The statement in the 2 nd page, 2 nd paragraph in the 2nd column, should be read as follows:

The output photoluminescence was collected using a $10-\mathrm{cm}$ lens and detected by a grating spectrometer or monochromator of $1 \AA$ spectral resolution (SPEX 750M), connected with a photomultiplier and a data scan [SPEX DS1010]. (Fig. 4a and 4b) The narrow emission bands presented in the article are detected again but from different samples indicating that they arise from the laser itself. Blue and blue-greenish upconversion luminescences are observed to the naked eye by excitation at $1046 \mathrm{~nm}$ of Nd:YAG laser pulses. Their pictures are presented below in Figs. $1 \mathrm{a}$ and $1 \mathrm{~b}$. The samples are located before the focus, where a breakdown in air is formed. Fig.1c pictures the samples' luminescence upon exposed to a UV lamp.

High photo-stability was again confirmed by continuously pumping the samples for two hours using a laser diode of photon energy $3.06 \mathrm{eV}$ and power $200 \mathrm{~mW}$. The detected output powers for both samples were decreased to an average of $\sim 65 \%$ of their initial values.

The online version of the original article can be found under doi:10.1007/s00340-012-4915-y.

I. M. Azzouz $(\bowtie) \cdot$ A. Salah

National Institute of Laser Enhanced Science, Cairo University,

Giza 12613, Egypt

e-mail: Iftazzouz@niles.edu.eg 

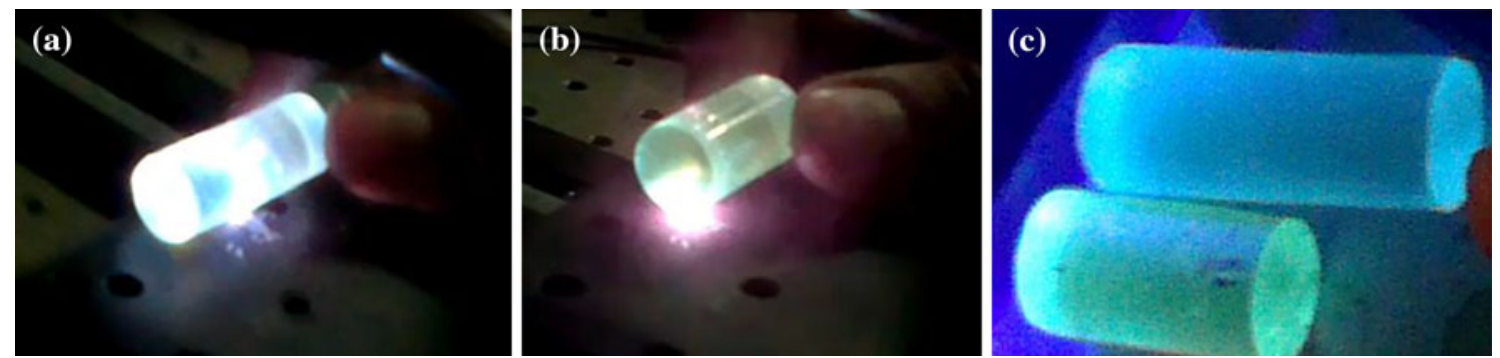

Fig. 1 a Blue and b blue-greenish upconversion luminescence by excitation at $1064 \mathrm{~nm}$ (air breakdown appeared as a bright spot below the samples). c Luminescence by a UV lamp 